\title{
The involvement of letter names in the silent processing of isolated letters: A developmental perspective
}

\author{
Paul Miller • Vered Vaknin
}

Published online: 5 September 2012

(C) Psychonomic Society, Inc. 2012

\begin{abstract}
This study was designed to clarify the nature of the mental representations underlying the processing of letters. A total of 96 Hebrew readers randomly recruited from three levels of education were asked to make rapid same/different judgments for Hebrew letter dyads with monosyllabic and bisyllabic names. The results obtained from the performance of participants under perceptual and conceptual processing conditions suggest that Hebrew readers access nominal letter representations in order to mediate letter processing in tasks that cannot be resolved on the basis of a sheer perceptual analysis of the letters' visual properties. The finding that the retrieval of nominal letter representations was evident for participants who differed rather markedly in their letter-processing speeds highlights the central role of letter names in the processing of isolated letters.
\end{abstract}

Keywords Psycholinguistics $\cdot$ Reading $\cdot$ Letter processing · Letter representations $\cdot$ Letter names

Letter graphemes are the basic units used by alphabetic orthographies to represent the phonemes of spoken words. It is therefore no surprise that, in current word-reading models, letter recognition is conceptualized as a distinctive processing module that in a sense precedes the psycholinguistic processes responsible for the recognition of the written words themselves (e.g., Coltheart, 1999; Grainger \& Jacobs, 1996; Jackson \& Coltheart, 2001; see also Finkbeiner \& Coltheart, 2009). The basic assumption of such models is that letters are recognized via mental representations that are insensitive to

P. Miller $(\bowtie) \cdot V$. Vaknin

Faculty of Education, University of Haifa,

Haifa, Israel

e-mail:mpaul@construct.haifa.ac.il the particularities of letters, such as their size, case, font, and color (e.g., A, a, $\mathcal{A})$.

Interestingly, however, different positions have been taken with regard to the nature of such representations. One position assumes that letter names serve as the critical category that mediates visual incongruity between letters (e.g., Posner \& Mitchell, 1967). In contrast, another position postulates this category to be of a more abstract, nonphonological nature (e.g., Kinoshita \& Kaplan, 2008). The present study was designed to further clarify the nature of the mental representations underlying the processing of decontextualized letters, by using a manipulation of the nominal syllabic properties of Hebrew letters.

The first systematic investigations of how readers process letters were conducted half a century ago by Posner and Mitchell (1967), who studied the role of visual and verbal (nominal) codes in letter recognition at different levels of processing with a series of letter-matching tasks. In one task, they asked participants to rapidly decide whether two letters were physically the same (e.g., A A) or different (e.g., A B). In a second task, the participants were required to classify letters as the same if they had the same name (e.g., A a) and as different if they did not (e.g., A b). Identicalness decisions for physically identical letters (e.g., A A) were found to be significantly faster in comparison to those to merely name-identical letters (e.g., A a). Posner and Mitchell concluded that in the first task participants could make a decision based upon the letters' perceptual properties, without the need to access a superordinate category - the letter names - whereas in the second task, they were forced to process the letters beyond a perceptual level; that is, they had to retrieve the letters' names in order to make identicalness decisions for pairs such as "A a." Note that identicalness decisions for identical letter pairs ("yes" responses) were faster than identicalness decisions for nonidentical 
letter pairs ("no" responses) (see also Miller, 2005; Soler \& Salvador, 1988). This "yes" response processing advantage makes particular sense in relation to the name-matching condition, in which making a "nonidentical" decision on the basis of differences in letters' visual properties was insufficient, given that such letters could still have the same name (e.g., a A).

Posner and Mitchell's findings (1967; see also Blake, Fox, \& Lappin, 1970; Posner \& Keele, 1967; Reitsma, 1978) suggest that the two processing conditions required different depths of processing: one in which processing was focused on the visual/perceptual properties of the letter stimuli, and another that involved the retrieval of some form of learned categorical knowledge, such as letter names. To further clarify the nature of the knowledge that mediates letter identicalness judgments, some researchers have manipulated the visual (e.g., E F) and phonological (e.g., P T) confusability of the letters comprising nonidentical stimulus pairs (Dainoff, 1970; Dainoff \& Haber, 1970; Thorson, Hochhaus, \& Stanners, 1976). The findings obtained from this line of research pointed to notably increased response latencies due to phonological and visual confusability between the nonidentical letters (Dainoff, 1970; Dainoff \& Haber, 1970; Thorson et al., 1976); that is, the findings supported an assumption that letter names are assigned a central role in the processing of decontextualized letters (e.g., Posner \& Mitchell, 1967; Proctor, 1981). A clarification of the role of letter names in the processing of letters was also a core issue in the present study.

In elementary school, novice readers often learn letters in close association with their names (e.g., Ellefson, Treiman, \& Kessler, 2009; Levin \& Aram, 2004), an association that is often stressed when letter names serve as a didactic means for bridging the visual incongruity between lowercase and uppercase letters or between print and cursive letters. Therefore, it makes sense to assume that the mediating potential borne by letter names is also exploited by individuals who are asked to determine letter identicalness under experimental conditions.

Interestingly, evidence from a series of studies conducted since the early 1980s has seemed to challenge this assumption. Carrasco, Kinchla, and Figueroa (1988) replicated both the Posner and Mitchell (1967) and the Thorson et al. (1976) studies with a sample of English- and Spanish-speaking university students. As expected, it took less time to determine that two physically identical letters (e.g., a a, A A) were the same than it took for two merely name-identical letters (A a). However, their findings failed to corroborate the finding that phonological confusability between nonidentical letters (e.g., b t from the Thorson et al., 1976, paradigm) impacted on "different" decisions, and this was found to be the case regardless of the size of the interstimulus interval. The absence of phonological interference in cross-case letter-matching tasks has also been noted in other studies manipulating the phonological similarity of letters in nonidentical pairs (Arguin \& Bub, 1995; Boles \& Eveland, 1983; Raynard \& Besner, 1987). In view of such massive counterevidence, a dual-code model that postulates the automatic activation of letter names in the processing of decontextualized letters (Posner \& Mitchell, 1967; see also Thorson et al., 1976) may not be tenable.

The nature of the code that individuals use to bridge the visual incongruity between name-identical letters in crosscase letter-matching paradigms was also studied by Kinoshita and Kaplan (2008). Their study incorporated three experimental conditions (Exps. 1-3), with each condition based on a manipulation of the "reference-prime-target" relationship on a specific case dimension (e.g., in Exp. 1, A-A-a [same] or D-A-a [different]; Exp. 2, A-a-a [same] or d-A-A [different]; Exp. 3, a-A-B [different]) and on a visual similarity dimension (e.g., Exp. 1, C-C-c [same]; Exp. 2, C-c-c [same]; Exp. 3, c-c-X [different]). The central research hypothesis was that, if the categorical knowledge that participants use to determine between-letter identicalness is abstract, manipulating the "reference-prime-target" relationship on a case dimension should have no impact on the size of the priming effect expected for identical letter dyads. This was predicted to be true even for letters that share notable visual similarity in different cases (e.g., $\mathrm{C}$ c). The robust priming effects reported by the researchers, in conjunction with a striking absence of bias from phonological or visual primetarget similarity, suggest that the participants processed the letter dyads down to abstract mental representations to determine their identicalness. The exact nature of such representations, however, remains obscure.

Additional insight regarding the exact nature of letter representations has been yielded from several lines of neuropsychological research investigating alexic readers who manifested an apparent inability to read letters and words, while manifesting surprisingly intact writing skills (e.g., Coslett \& Saffran, 1992; Saffran \& Coslett, 1998). Moreover, Mycroft, Hanley, and Kay (2002) reported that a pure alexic patient (M.S.) with no access to letter names nevertheless manifested normal ability to visually discriminate between letters, to match between identical letters presented in different cases (cross-case matching), and to search for specific letters from within letter sequences. Taken together, M.S.'s performance pattern suggests access to representations that encode letters as specific visual objects. Moreover, the patient also seemed capable of referencing specific abstract letter identities that do not reflect the visual features of the letters (see Miozzo \& Caramazza, 1998). These findings actually suggest that letter names may not directly mediate the processing of letters.

The majority of studies testing hypotheses regarding the way that letters are processed have done so in orthographiessuch as English-whose letters lack discriminating inherent 
properties (e.g., variance in letter name syllabic length) that would allow for an examination of the involvement of letter names in the processing of decontextualized letters in a straightforward manner. As a consequence, conclusions drawn from such studies have often failed to directly corroborate hypotheses concerning the nature of the letter representations (e.g., abstract nominal, abstract visual) that readers retrieve in order to determine mono-case (e.g., A A, a a, A B, a b) and cross-case (e.g., A a, B a) letter identicalness. This may explain why replications of seminal studies in the domain of letter processing (e.g., Posner \& Mitchell, 1967) have yielded conflicting findings. It may also explain why questions regarding the nature of letter representations and the way that these representations mediate the processing of isolated letters have not yet received decisive answers.

The innovation of the present study is that it drew on the inherent discriminating features of Hebrew letters - namely, the syllabic length of the letter name (hereafter, "syllabic length") - to determine the nature of letter representations and the way that they impact the processing of letters at different depths (see below). Unlike most other orthographies, Hebrew letters have either monosyllabic or bisyllabic names $^{1}$ (see Levin, 2007). Taking advantage of this fact, we asked participants to make rapid same/different judgment for letter dyads simultaneously presented on a laptop display (Posner \& Mitchell, 1967, paradigm), with half of the pairs built of two letters with monosyllabic names and the remainder of two letters with bisyllabic names. The participants were tested with the same stimulus list under two experimental conditions. In the perceptual condition ( $P$ condition), the two letters in the pair were always presented in the same type of script (exemplified in English as A A, $\boldsymbol{a} \boldsymbol{a}, \mathrm{A} \mathrm{B}, \boldsymbol{a} \boldsymbol{b})^{2}$ In the conceptual condition ( $C$ condition), one letter in the pair was always presented in print and the other in cursive script (exemplified in English as A $\boldsymbol{a}, \mathrm{A} \boldsymbol{b}$ ). In order to examine developmental trends in the way that syllabic length impacts letter recognition at different depths of processing, we tested readers from ascending educational levels.

The impetus underlying this study was to clarify whether letter names provide the basis for the formation of the representations that readers use to mediate the processing of isolated letters, as hypothesized by Posner and Mitchell (1967). We further were interested in whether the processing

\footnotetext{
${ }^{1}$ Greek is another orthography composed of letters with mono- and bisyllabic names (e.g., $\pi=$ pi [monosyllabic], $\beta=$ beta [bisyllabic]). However, unlike Greek letters, which bear strong associations with mathematical concepts, Hebrew letters are semantically neutral.

${ }^{2}$ Unlike English letters, Hebrew letters are not realized as uppercase and lowercase; therefore, we used print and cursive letters instead. Moreover, in Hebrew, the print and cursive scripts are two systems used for different functions. Note that, unlike uppercase and lowercase letters, the two systems do not appear regularly in the same text.
}

of isolated letters triggers their names, even in instances in which this is not necessary for task performance. In short, our aim was to determine the involvement of letter names in the mediation of letter identicalness at different levels of processing. Finally, we considered whether the strategies that readers rely on to process isolated letters are modified by increased reading experience.

\section{Research hypotheses}

Several possible forms of mental representations (categories) have been suggested in the literature as underlying the processing of isolated letters: (1) abstract visual representations that depict the critical visual features of letters (e.g., Mycroft et al., 2002; Posner \& Mitchell, 1967); (2) abstract phonemic representations that represent the critical phonological characteristics of how letters are pronounced (e.g., Carrasco et al., 1988); (3) abstract nominal representations - that is, letter names (e.g., Posner \& Mitchell, 1967); and (4) abstract non-code-specific representations that reflect the convention that two different letter shapes (e.g., A a) are the same letter (e.g., Kinoshita \& Kaplan, 2008; Miozzo \& Caramazza, 1998). In order to contribute to a better understanding of how isolated letters are processed, we tested several hypotheses:

A. From the assumption that readers rely on nominal representations (i.e., letter names) to process isolated letters, it was hypothesized that - in general - it would be notably faster to determine the identicalness of letters with monosyllabic names than of those with bisyllabic names (syllabic effect). We expected this to be true in relation to both identical and nonidentical letter pairs. This was because in order to make a decision about letter pairs composed of bisyllabic letters, participants would have to process twice as much phonological information as would be necessary to make the same decision for letter pairs composed of monosyllabic letters.

B. In order to determine the identicalness of two physically distinct, yet conventionally identical letters (e.g., A a), the time-consuming retrieval of some form of superordinate category that bridges the visual incongruity between the stimuli would be a prerequisite. In contrast, to determine the identicalness of two physically identical letters (e.g., A A), an analysis of their perceptual properties would, in principle, be sufficient. We therefore hypothesized that participants would take significantly less time to determine letter identicalness under the $\mathrm{P}$ condition than under the $\mathrm{C}$ condition.

C. Assuming that an analysis of perceptual properties is enough to determine the identicalness of two physically 
identical letters, we hypothesized that syllabic length would impact letter processing under the $\mathrm{C}$ condition but not the $\mathrm{P}$ condition.

D. Finally, we hypothesized that faster letter processing would lead to reduced bias from syllabic length under the $\mathrm{C}$ condition. This is because readers - due to enhanced reading experience that results in enhanced automation in letter recognition - gradually move from reliance on nominal representations at the initial stages of reading acquisition to more letter-sound-based representations that underlie grapheme-to-phoneme conversion processes (see Treiman \& Rodriguez, 1999; Treiman, Sotak, \& Bowman, 2001).

\section{Method \\ Participants}

The participants were 96 students randomly recruited from schools in northern Israel from three levels of education. They included 30 third and fourth graders (elementary school level $=$ EL), 37 sixth and seventh graders (middle school level $=$ ML), and 29 ninth and tenth graders (high school level $=\mathrm{HL}$ ). All of the participants had normal or corrected-to-normal vision. According to their school files, their intelligences were considered normal. Only individuals not diagnosed as having a history of hearing impairments, learning disabilities, or emotional disorders were included in the study.

\section{Stimuli and design}

Paradigm description To examine the developmental aspects related to the involvement of letter names in the processing of isolated letter stimuli, we implemented a 2 (syllabic length: monosyllabic or bisyllabic) $\times 2$ (depth of processing: physically identical or conventionally identical) $\times 3$ (educational level: EL, ML, or HL) mixed design. We used an elaborated version of the Posner and Mitchell (1967) paradigm in which we asked participants to make rapid same/different judgments for a series letter pairs presented one after the other in the center of a computer display. The letters in a pair were separated from each other by $3.5 \mathrm{~cm}$, a distance that prevented focusing on both of them at the same time. The participants were asked to press a key marked YES for pairs of identical letters and a key marked NO for pairs of nonidentical letters.

A set of 40 letter pairs was used as stimuli. Twenty of these pairs were composed of letters with monosyllabic names, and the remainder of letters with bisyllabic names. In each syllabic category, half of the pairs (ten) contained the same letter twice (exemplified in English as B B), while the other half consisted of two different letters (exemplified in English as B T). In order to compare depths of processing, the 40 stimulus pairs were prepared in two versions - perceptual and conceptual-with each version providing a specific experimental condition.

In the $\mathrm{P}$ condition, both letters in the pair were presented in the same script, either print or cursive. Consequently, in pairs composed of the same letter twice, the letters were physically identical ( $\boldsymbol{\beth}$ ב or $\boldsymbol{\supset} \boldsymbol{\curvearrowright}$ exemplified in English as b b or $\boldsymbol{b} \boldsymbol{b}$ ) and shared the same name. In nonidentical pairs, the letters were physically different and had different names, but were presented in the same script type ( $\boldsymbol{Z} \boldsymbol{\Delta}$ or $\boldsymbol{\nabla} \boldsymbol{N}$, exemplified in English as b r or $\boldsymbol{l} r$ ).

In the $\mathrm{C}$ condition, one letter of the pair was presented in print and the other in cursive script. Thus, in pairs composed of a repeated letter, the two were no longer physically identical ( $\boldsymbol{\supset} \boldsymbol{D}$, exemplified in English as $b \boldsymbol{b}$ ), but they shared the same name. In nonidentical letter pairs, each letter also appeared in a different script type ( $\supset$ ฉ, as exemplified in English $\mathrm{b} \boldsymbol{r}$ ), and they had different names.

Stimulus preparation procedure The Hebrew alphabet contains 22 letter graphemes, with some of them implemented in a different shape when appearing at the end of a word. Sixteen of them have monosyllabic names (e.g., $ב=$ bet), and six have bisyllabic names (e.g., $\lambda=$ gimel). To build the stimulus pairs used for experimentation, we chose from each category five letters with the most distinct shapes in print and cursive script (Appendix A).

To prepare the dyads for the $\mathrm{P}$ condition, we first created five physically identical pairs with monosyllabic names (e.g., ב ב ב ב בet bet]) and five with bisyllabic names (e.g., ג $\lambda$ [gimel gimel]). We then used the same letters to prepare ten nonidentical letter pairs, half built from monosyllabic letters (e.g., מ ב ב ב ב ב letters (e.g., ל ל ל ב ל ב versions of the 20 letter pairs - one in print and another in cursive script - to arrive at a total of 40 stimulus pairs (Appendix A).

To prepare the stimulus pairs for the $\mathrm{C}$ condition, we used the same 40 pairs created for the $\mathrm{P}$ condition, but changed the script type, so that one letter was always presented in print and the other in cursive script (e.g., $\boldsymbol{\beth} \boldsymbol{D}=$ conceptually identical; $\boldsymbol{\Delta} \boldsymbol{\boldsymbol { D }}=$ different letters) (Appendix A). Nonetheless, the proportions of letters in print and in cursive in the two conditions remained exactly the same, allowing for direct comparisons between the conditions.

Letter frequency To test for possible letter frequency differences between the monosyllabic and bisyllabic letters we used, we counted the occurrence of each of them in a total of 8,841 words taken from 15 story texts (five at the elementary level, five at the middle school level, and five at the 
high school level) and five electronic news articles from the Internet. The average frequencies were $3.39 \%$ for the monosyllabic letters and $3.38 \%$ for the bisyllabic letters (Appendix B).

\section{Procedure}

Each participant was tested individually. We used the DMDX software developed by K. I. Forster and J. C. Forster (2003) for stimulus presentation and data collection. This technology enables the measurement of response latencies in the range of milliseconds.

Task explanation Participants were asked to sit in front of a laptop used to present the test stimuli. They were told to place their index fingers onto the two Shift keys: The right one was labeled YES, and the left one labeled NO. The experimenter informed the participants that they would see two letters simultaneously in the center of the monitor and instructed them to press YES when the two letters were the same and $\mathrm{NO}$ when the letters were different. He then displayed ten practice pairs, one after another, to test the participants' understanding of the task requirements. None manifested problems in comprehending the task requirements.

Assessment of letter-processing efficiency Prior to beginning the stimulus presentation, the experimenter told participants to indicate their response as quickly as possible, because time was being measured. The 40 letter pairs of the $P$ condition and the 40 pairs of the $C$ condition were presented in two separate blocks, rotated alternately between the participants, with presentation controlled by the computer. Each letter presentation was preceded by the characters \#\#\#\# in order for the participants to focus on the display location. Each pair was displayed until participants had indicated their decisions by pressing a response key (YES or NO), but not for longer than $3,500 \mathrm{~ms}$. The interstimulus presentation interval was $500 \mathrm{~ms}$. Both reaction time (speed of processing) and decision accuracy (processing error rate) were recorded for future analysis.

\section{Results}

The error rates were generally low (fewer than two errors out of 80 possible). We therefore decided to restrict the analyses to the speed of letter processing only.

The basic assumption for choosing participants of different education levels was that in the course of education, processing letters and written words becomes increasingly automatized; educational experience such as learning to read accelerates access to this knowledge. To determine whether participants' education level was indicative of their processing speed, we divided them - in addition to their original specific levels of education-into three response speed clusters: slow, medium, and fast. This clustering was based on their overall response latencies: Participants 3/4 of an $S D$ above the sample average were assigned to the slow cluster, those 3/4 of an $S D$ below the average to the fast cluster, and the remainder to the medium cluster.

To make a final decision on which of the two betweensubjects factors was most adequate for the investigation of the syllabic length effect, we conducted a CrossTab analysis that elucidated the degree to which the participants of specific education levels overlapped participants assigned to the three response speed clusters. As can be seen in Table 1, although participants from ascending grade levels are predominately assigned to descending response speed clusters, this overlap is not entirely matched. This seems to suggest that experience leading to increased automation of letter processing and reduced bias from syllabic length (Hypothesis D) may not be intrinsically linked to the individual's level of education. Therefore, we decided to use response speed (slow, medium, high) rather than level of education as the betweensubjects factor and to compute education level as a covariate in subsequent analyses.

We applied a linear mixed-effects model procedure to our data to account for the correlation within subjects. In this analysis, we computed Syllabic Length (monosyllabic, bisyllabic), Depth of Processing (perceptual, conceptual), and Response Type (identical, nonidentical) as fixed within-subjects factors, Response Speed Cluster (slow, medium, fast) as the between-subjects factor ( 80 observation for each of the 96 participants), and education level (elementary, medium, high) as a covariate. The $p$ values of the comparisons among the main effects were adjusted to allow for multiple comparisons by applying the Bonferroni procedure.

Table 2 presents the mean reaction times (RTs) for monosyllabic (MO) and bisyllabic (BI) letters under the P and C conditions overall, and separately for each response speed cluster.

Table 1 Degree of concordance between the participants' level of education and their response speed cluster

\begin{tabular}{lcccc}
\hline \multirow{2}{*}{ Level of Education } & \multicolumn{2}{l}{ Type of Responder } & \multirow{2}{*}{ All } \\
\cline { 2 - 4 } & Slow & Medium & Fast & \\
\hline EL & 20 & 10 & 0 & 30 \\
ML & 13 & 16 & 8 & 37 \\
HL & 0 & 11 & 18 & 29 \\
All & 33 & 37 & 26 & 96 \\
\hline
\end{tabular}

EL, elementary school level; ML, middle school level; HL, high school level 
Table 2 Adjusted SoP means (in ms) for mono - and bisyllabic Hebrew letters under P- and C-conditions, by response type (SEs in parentheses) Response Type

\begin{tabular}{|c|c|c|c|c|c|c|c|}
\hline \multirow[b]{2}{*}{$\mathrm{DP}^{\mathrm{a}}$} & \multirow[b]{2}{*}{$\mathrm{Gr}^{\mathrm{b}}$} & \multicolumn{2}{|l|}{ Identical } & \multicolumn{2}{|l|}{ Nonidentical } & \multicolumn{2}{|l|}{ Overall } \\
\hline & & MO & BI & MO & BI & MO & BI \\
\hline \multirow[t]{4}{*}{$\mathrm{p}^{\mathrm{c}}$} & $\mathrm{S}$ & $870(20.33)$ & $852(20.36)$ & $1100(20.40)$ & $1000(20.40)$ & $985(17.62)$ & $926(17.63)$ \\
\hline & M & $653(19.31)$ & $640(19.27)$ & $753(19.40)$ & $706(19.16)$ & $703(16.71)$ & $673(16.63)$ \\
\hline & $\mathrm{F}$ & $552(22.87)$ & $522(22.92)$ & $598(23.05)$ & $580(22.91)$ & $575(19.86)$ & $551(19.83)$ \\
\hline & A & $692(12.06)$ & $672(12.07)$ & 817 (12.13) & $762(12.07)$ & $754(10.46)$ & 717 (10.44) \\
\hline \multirow[t]{4}{*}{$C^{d}$} & $\mathrm{~S}$ & 1154 (20.68) & $1227(21.02)$ & $1265(20.59)$ & $1350(20.60)$ & $1210(17.78)$ & $1289(17.88)$ \\
\hline & M & $822(19.58)$ & 889 (19.76) & 917 (19.58) & 909 (19.34) & $869(16.85)$ & 899 (16.23) \\
\hline & $\mathrm{F}$ & $655(23.35)$ & $706(23.43)$ & $725(23.20)$ & $713(23.08)$ & $690(20.04)$ & $710(20.03)$ \\
\hline & A & 877 (12.28) & $941(12.39)$ & $969(12.23)$ & 991 (12.16) & $923(10.55)$ & $966(10.56)$ \\
\hline \multirow[t]{4}{*}{ Tot. } & $\mathrm{S}$ & $1011(17.70)$ & $1039(17.81)$ & $1183(17.70)$ & $1175(17.70)$ & $1097(16.12)$ & $1107(16.12)$ \\
\hline & M & 737 (16.77) & $765(16.81)$ & 835 (16.79) & 807 (16.65) & $786(15.25)$ & $786(15.23)$ \\
\hline & $\mathrm{F}$ & $604(19.95)$ & $614(19.98)$ & $661(19.96)$ & $647(19.88)$ & $633(18.16)$ & $630(18.51)$ \\
\hline & A & $784(10.50)$ & $806(10.54)$ & $893(10.51)$ & $876(10.47)$ & $838(9.56)$ & $841(9.56)$ \\
\hline
\end{tabular}

${ }^{\mathrm{a}}$ Depth of processing; ${ }^{\mathrm{b}}$ Group: $\mathrm{S}=$ Slow responders, $\mathrm{M}=$ Medium responders, $\mathrm{F}=$ Fast responders; ${ }^{\mathrm{c}}$ Perceptual processing condition; ${ }^{\mathrm{d}}$ Conceptual processing condition

The syllabic length effect (3 ms) was not statistically significant, $F(1,7105.787)=0.18, p>.05$; that is, processing of monosyllabic letter pairs $(M=838, S E=9.56)$ took as long overall as processing bisyllabic letter pairs $(M=841$, $S E=9.56$; see Table 2). The main effect of depth of processing ( $\mathrm{P}$ condition, $\mathrm{C}$ condition) was statistically significant, $F(1,7106.303)=1,151.77, p<.001$; determining letter identicalness was markedly faster under the $\mathrm{P}$ condition $(M=736, S E=9.53)$ than under the $\mathrm{C}$ condition $(M=$ $944, S E=9.59)$. The main effect of response type (identical, nonidentical) was also statistically significant, $F(1$, $7106.110)=211.45, p<.001$, implying that participants judged identical letter pairs faster $(M=795, S E=9.57)$ than nonidentical letter pairs $(M=884, S E=9.55)$. As expected, the response speed cluster main effect was statistically significant, $F(2,92.723)=227.02, p<.001$. Bonferroni posthoc analysis confirmed that the average response speeds of the three clusters were significantly different (slow, $M=1,102, S E=15.27$; medium, $M=786, S E=14.43$; fast, $M=631, S E=17.20)($ all $p \mathrm{~s}<.001)$.

The linear mixed-effects model procedure revealed several interactions between fixed effects. The interactions reported next are restricted to the way that the letter name syllabic-length effect - the target factor of this study - interacted with the other main effects - namely, depth of processing, response type, and response speed cluster.

The analysis revealed a significant interaction between syllabic length and depth of letter processing, $F(1,7106.306)=$ $42.66, p<.001$, implying that syllabic length biased the speed of processing differently under the $\mathrm{P}$ and $\mathrm{C}$ conditions. As can be seen in Table 2, under the $\mathrm{P}$ condition the syllabic length effect $(-37 \mathrm{~ms})$ was contrary to the predicted direction, with bisyllabic letter pairs being processed faster than monosyllabic letter pairs. In contrast, under the $\mathrm{C}$ condition, the syllabic length effect was in the predicted direction (43 ms), with monosyllabic letter pairs being processed faster than bisyllabic letter pairs. Note that the absence of a significant threeway interaction - Syllabic Length $\times$ Depth of Processing $\times$ Response Type, $F(2,7105.848)=0.09, p>.05$-further indicated that overall, similarly inverse syllabic-length effects for the $\mathrm{P}$ and $\mathrm{C}$ conditions were also evident when the impact of syllabic length on each of the two response types was considered (see Table 2).

The analyses further revealed a significant interaction between syllabic length and response type, $F(1,7105.856)=$ 9.77, $p<.01$, indicating that syllabic length biased the processing of identical and nonidentical letter pairs differently. As can be seen in Table 2, the two response conditions produced inverse syllabic-length effects: Monosyllabic letter pairs were processed faster than bisyllabic letter pairs $(22 \mathrm{~ms})$ in identical letter pairs ("yes" response), and monosyllabic letter pairs were processed slower than bisyllabic pairs $(-17 \mathrm{~ms})$ in nonidentical letter pairs ("no" response). The nonsignificant threeway interaction Syllabic Length $\times$ Response Type $\times$ Response Speed Cluster, $F(2,7105.850)=0.51, p>.05$, further indicates that similarly inverse syllabic-length effects for "yes" and "no" responses were also found for the three response speed clusters separately (see Table 2). 
The interaction between the main effect of syllabic length and the response speed cluster main effect was not statistically significant, $F(2,7105.787)=0.38, p>.05$, suggesting that overall, syllabic length biased letter-processing speeds in the three response speed clusters uniformly (see Table 2). However, a statistically marked three-way interactionSyllabic Length $\times$ Depth of Processing $\times$ Response Speed Cluster, $F(2,7106.295)=5.70, p<.01$-indicated that the size of the syllabic-length effect under the $\mathrm{P}$ and $\mathrm{C}$ conditions was not uniform for the three response speed clusters. As can be seen in Table 2, in both the $\mathrm{P}$ and the $\mathrm{C}$ conditions, bias due to syllabic length decreased with increases in letter-processing speed. Of particular interest in this regard is the $\mathrm{C}$ condition, in which slow responders manifested a syllabic effect more than double $(79 \mathrm{~ms})$ that manifested by medium or fast responders (30 or $20 \mathrm{~ms}$, respectively). Moreover, in contrast to medium and fast responders, slow responders exhibited a remarkable syllabic effect $(85 \mathrm{~ms})$ when processing nonidentical letter pairs under the $\mathrm{C}$ condition (see Table 2). This is in contrast to the other two response speed clusters, which for the same letter pairs exhibited only small and inverted syllabic effects.

\section{Discussion}

This study aimed to shed further light on the nature of the mental representations that readers use to mediate the processing of isolated letters. The main findings from this study indicated that Hebrew letters with bisyllabic names are processed, overall, markedly slower in comparison to Hebrew letters with monosyllabic names. Interestingly, however, evidence suggests that syllabic length impacts processing mainly in instances that require conceptual processing.

The discussion in the following paragraphs will emphasis the impact of syllabic length on the processing of identicalletter dyads rather than nonidentical-letter pairs, as effects related to letter name in nonidentical dyads are at risk of being confounded with effects originating from factors such as letter frequency, visual letter confusability (Finkbeiner \& Coltheart, 2009), phonological letter neighborhood similarity, and letter combination likelihood. We will refer to the processing of nonidentical-letter dyads only when their analysis helps explain how syllabic length impacts participants' quantitative processing behavior.

As stated earlier, several potential candidates can take on a mediating function in the processing of isolated letters, such as abstract visual (e.g., Mycroft et al., 2002; Posner \& Mitchell, 1967), abstract phonological (e.g., Carrasco et al., 1988), abstract nominal (e.g., Posner \& Mitchell, 1967), and/or abstract non-code-specific (e.g., Kinoshita \& Kaplan,
2008; Miozzo \& Caramazza, 1998) representations, each of which has received some support in the literature. On the other hand, teachers and parents tend to refer to letters in a nominal way as they introduce them to their "novice readers/writers" (Levin \& Aram, 2004; Treiman \& Rodriguez, 1999; Treiman et al., 2001), with preschoolers being more acquainted with letter names than with their sounds (Levin, Shatil-Carmon, \& Asif-Rave, 2005). Assuming that letter names continue to play a central role in mediating the processing of letters even among proficient readers therefore makes sense (Dainoff, 1970; Dainoff \& Haber, 1970; Posner \& Mitchell, 1967; Proctor, 1981; Treiman et al., 2001). Interestingly, much previous evidence has seemed to contest this assumption (Arguin \& Bub, 1995; Boles \& Eveland, 1983; Carrasco et al., 1988; Kinoshita \& Kaplan, 2008; Raynard \& Besner, 1987). The method used in the present study allowed for a direct corroboration of the involvement of letter names in the processing of isolated letters by comparing the processing of monosyllabic and bisyllabic dyads.

If readers rely on mental representations that are nominal in nature (letter names) to process isolated letters, it will be notably faster to determine the identicalness of letters with monosyllabic names than of those with bisyllabic names (Hypothesis A from the introduction). The findings of the present study indeed indicate that the amount of syllabic information in letter names markedly biased speed of processing in the $\mathrm{C}$ condition (Tables 2). This contradicts evidence suggesting that letter names do not play a vital role in the processing of isolated letters (Arguin \& Bub, 1995; Boles \& Eveland, 1983; Carrasco et al., 1988; Kinoshita \& Kaplan, 2008; Mycroft et al., 2002; Raynard \& Besner, 1987). Of particular interest in this regard is how variation in syllabic length impacted on determining whether two visually dissimilar but conventionally identical letters were the same. A detailed analysis of "yes" responses in the $\mathrm{C}$ condition pinpointed rather remarkably delayed response latencies (64 ms) for bisyllabic letter dyads (ג C [gimel gimel]) in comparison to monosyllabic letter dyads (e.g., ح [bet bet]) (Table 2). Given that, in this instance, bias originating from syllabic length was not at risk of being confounded with interference from competing effects (see above), in conjunction with the fact that prominent syllabic-length effects were found for all three response speed clusters (slow, medium, and fast) and, overall, also with regard to nonidentical letter dyads, this evidence is particularly noteworthy. It supports the conclusion that our participants retrieved the letters' names in the course of determining letter identicalness, a conclusion that is in line with a position (Posner \& Mitchell, 1967) that assigns letter names a mediating function in the processing of merely conventionally identical letters.

A basic assumption in this study was that, in order to determine the identicalness of two physically distinct yet 


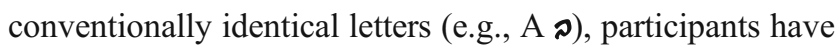
to access some form of categorical knowledge in a timeconsuming process to bridge the visual incongruity between the letter stimuli. We further assumed that this may not be necessary to determine the identicalness of two physically identical letters (e.g., A A or $\boldsymbol{a} \boldsymbol{a}$ ), in which case a visual analysis of the stimuli may be sufficient. We consequently predicted (Hypothesis B) that participants would take significantly less time to determine letter identicalness under the $\mathrm{P}$ condition than under the $\mathrm{C}$ condition. A statistically highly significant depth-of-letter-processing effect corroborated this hypothesis; that is, making identicalness decisions under the $\mathrm{P}$ condition was indeed remarkably faster (736 ms) than making them for the same letter dyads under the $\mathrm{C}$ condition $(944 \mathrm{~ms})$. This was true for all three response speed clusters and regardless of response type ("yes," "no") or syllabic length (monosyllabic, bisyllabic) (Table 2). Given such convergent evidence, it makes intuitive sense to conclude that, in order to make an identicalness judgment under the $\mathrm{P}$ condition, participants relied on the sheer perceptual analysis of the letters' visual properties. Such a conclusion would also be in line with a position (dual-code model; Posner \& Mitchell, 1967) that considers visual and phonological coding as two independent, consecutive processing stages that may become subject to mental operations, such as making a letter identicalness decision.

Markedly decreased response latencies in the $\mathrm{P}$ condition give rise to a theory that an analysis of perceptual/visual properties is in principle sufficient to determine the identicalness of two physically identical letters (e.g., A A). To substantiate the validity of this theory, we tested whether syllabic length impacted on letter processing under the $\mathrm{C}$ condition but not the $\mathrm{P}$ condition, as suggested by Hypothesis C. A statistically significant interaction between letter syllabic length and depth of processing revealed by mixed-model analysis indeed indicated that syllabic length biased letter processing under the $\mathrm{P}$ and $\mathrm{C}$ conditions differently, pointing to a syllabic-length effect under the $\mathrm{C}$ condition but not under the $\mathrm{P}$ condition (Table 2). This proved true for all three response speed clusters. Interestingly, as seen in Table 2, processing letter pairs under the $\mathrm{P}$ condition produced an inverse syllabic length effect: monosyllabic letter pairs were processed more slowly than bisyllabic letter pairs (e.g., ב ב [bet bet] ג ג vs. [gimel gimel]).

The absence of a "true" syllabic length effect under the $\mathrm{P}$ condition deserves further consideration. It actually suggests that at this early stage of processing, readers may not pay substantial attention to letters' nominal information for task performance, but concentrate more on a task-adequate analysis of the letters' physical properties. The possibility that the visual analysis of letters' properties dominated the processing of letter identicalness under the $\mathrm{P}$ condition is indirectly supported by the inverse syllabic length effect found in this condition in relation to both identical and nonidentical letter pairs. The following two explanations consider this possibility in more detail.

A close look at the letters used to create nonidentical letter pairs shows that letters in nonidentical monosyllabic letter dyads - although visually distinct — were overall visually much more similar than those used for building the bisyllabic letter dyads (Appendix A). In other words, due to increased visual similarity, hence visual confusability (see Finkbeiner \& Coltheart, 2009; James, Wong, \& Jobard, 2010), it was probably harder to reject the identicalness of nonidentical monosyllabic than of bisyllabic letter pairs. So, it seems reasonable to assume that the inverse syllabic-length effect found under the $\mathrm{P}$ condition in fact reflects a visual similarity effect originating from increased letter confusability (see, e.g., Treiman, Levin, \& Kessler, 2006); that is, it is a byproduct of the intensified visual processing of the stimulus pairs.

Interpreting inverse syllabic length effects in the $\mathrm{P}$ condition purely as visual (perceptual) similarity effects, which is convincing for nonidentical letter pairs, should be applied with some caution when explaining the inverse syllabiclength effect found in the processing of identical letter pairs in the P condition. In fact, finding inverse syllabic-length effects in both response conditions suggests that an explanation referring merely to the visual dimension of letters may be too simplistic, and has to be expanded to include some level of letter knowledge. This is because reliance on sheer visual processing should not have biased the processing of physically identical letter pairs, whether they had mono- or bisyllabic names. In other words, the finding of such a bias suggests that although they are principally redundant for task performance, some form of letter representations under the $\mathrm{P}$ condition that did not reflect syllabic length was accessed by participants. Such representations - probably of an abstract, visual nature - were likely to trigger neighborhood letter representations with overlapping abstract visual properties.

Indeed, a close examination of the visual properties of the letters used for building identical monosyllabic letter pairs, as opposed to the letters used for the preparation of identical bisyllabic letter pairs, with reference to other Hebrew letters with overlapping visual properties (e.g., ב, つ, ר), shows that such overlapping was more prevalent for monosyllabic letters (see Appendix A). Provided that this prevalence is also reflected in the letters' abstract visual representations, it makes sense to assume that the encounter with monosyllabic letters under the $\mathrm{P}$ condition triggered more potentially competing neighborhood letter representations than did the encounter with bisyllabic letters. This 
would reasonably explain why inverse syllabic-length effects were also found for identical letter pairs under this condition.

A question of central interest is, of course, whether increases in letter-processing speed modify the nature of the representations that mediate the processing of isolated letters at different depths of processing. For example, it may be possible that readers who-most likely as a consequence of more reading experience-become faster at letter processing move from a nominal letter-processing strategy to the use of representations of a more abstract, noncode-specific nature that reflect the convention that two different letter shapes are the same letter (e.g., A a) (Finkbeiner \& Coltheart, 2009; Kinoshita \& Kaplan, 2008) or that two visually distinct letter shapes represent the same phoneme (see Treiman \& Rodriguez, 1999; Treiman et al., 2001). Assuming this to be true, we predicted that an increase in the speed of letter processing would lead to a parallel decrease in the size of the syllabic-length effect under the $\mathrm{C}$ condition (Hypothesis D).

Quite expectedly, and in line with previous research (e.g., Miller, 2005; Reitsma, 1978), identicalness judgments under the $\mathrm{C}$ condition became significantly faster as a function of increased reading experience (slow, $M=1,102 \mathrm{~ms}$; medium, $M=786 \mathrm{~ms}$; fast, $M=631 \mathrm{~ms}$ ). This was true in relation to the processing of both identical- and nonidentical-letter dyads, regardless of syllabic length (Table 2). Of note, the findings from the present study point to a rather marked decrease in the overall syllabic-length effect manifested by the medium and fast responders (30 and $19 \mathrm{~ms}$, respectively), in comparison to the slow responders $(78 \mathrm{~ms})$, and to a gradual decrease in relation to identical responses (slow, $71 \mathrm{~ms}$; medium, $68 \mathrm{~ms}$; fast, $50 \mathrm{~ms}$ ). In line with Hypothesis $\mathrm{D}$, such a decrease in the syllabic-length effect may indicate a shift from the predominant involvement of nominal representations in the processing of letters at the initial stages of reading acquisition (see Table 2) to reliance on more lettersound-based representations underlying grapheme-tophoneme conversion processes as reading becomes more automatized (see Treiman \& Rodriguez, 1999; Treiman et al., 2001). Alternatively, the decrease of the syllabic-length effect under the $\mathrm{C}$ condition may indicate that for moreproficient letter processors, the encounter with letters-in addition to nominal letter representations - simultaneously triggers letter representations of a more abstract, phonological (see Treiman \& Rodriguez, 1999; Treiman et al., 2001), or non-code-specific (Finkbeiner \& Coltheart, 2009; Kinoshita \& Kaplan, 2008) nature. Such representations are likely to assume a mediating function, hence leading to a reduced processing bias due to letters' nominal properties.

It is noteworthy that, even after such a remarkable decrease overall, the syllabic-length effect remained robust when participants had to determine the identicalness of merely conventionally identical letter pairs (C condition). Given that this condition was hypothesized to pinpoint syllabic-length effects most purely, it can be concluded that letter names continue to play a central role even for experienced readers. In other words, the introduction of more advanced letter-processing strategies does not necessarily lead readers to abandon earlier processing strategies (e.g., Rittle-Johnson \& Siegler, 1999; Treiman et al., 2001), as long as these strategies prove efficient for task performance.

In summary, the syllabic-length effects found in the present study suggest that nominal letter representations are involved in the processing of decontextualized Hebrew letters in instances that require their processing beyond a perceptual level (C condition). These findings challenge conclusions drawn from studies that have seemed to refute the involvement of letter names in the processing of isolated letters (Arguin \& Bub, 1995; Boles \& Eveland, 1983; Carrasco et al., 1988; Kinoshita \& Kaplan, 2008; Raynard \& Besner, 1987; see also Finkbeiner \& Coltheart, 2009).

As stated earlier, determining the identicalness of two physically identical letters does not, in principle, require the retrieval of letter names or of any other kind of mental representations (abstract visual, phonological, or non-codespecific) to mediate the identicalness of the stimuli. Theoretically, this could be accomplished even by someone totally unfamiliar with the stimulus materials themselves and could be based on a purely visual analysis of the stimuli (e.g., $\lambda \lambda$ ). The finding of significant inverse syllabic effects in "yes" and "no" responses under the P condition seems to contradict this assumption. It actually suggests that whereas letter names may not hold a mediating function in tasks that can be resolved by the visual processing of the stimuli, representations other than nominal letters may well be involved. This may lead to the conclusion that once readers have internalized knowledge of letters, this knowledge automatically operates in their encounters with decontextualized letters, though different types of letter knowledge may dominate at different depths of processing. This conclusion is in line with a more general theory of visual object recognition; namely, conceptual information appears to be recruited even for tasks traditionally deemed primarily perceptual (T. W. James \& Cree, 2010, p. 343).

The findings from the present study seem to suggest that abstract representations of letters' perceptual properties are activated at a relatively early stage of letter processing, with nominal representations coming into play at later, more profound levels of processing. Thus, a question to be resolved is whether the processing of familiar, decontextualized letters, at any point, can be purely visual in nature. To 
answer this question, future research should compare readers unfamiliar with Hebrew letters with native Hebrew readers under the $\mathrm{P}$ condition. Findings from such research might indicate whether the inverse syllabic effects yielded in this condition indeed indicate the involvement of abstract, nonnominal letter representations in the initial stage of processing.

The majority of more recent studies (for a summary, see Finkbeiner \& Coltheart, 2009) focusing on the earliest stage of reading - the perception of letters - have primarily examined how letters are extracted from visual features and how such features are reflected in abstract mental representations, with minimal attention given to the role of nominal information in the processing of the decontextualized letters used for experimentation. Our findings suggest that such disregard for nominal representations may not be justified, as letter names seem to become available to the reader in the processing of letters and, consequently, may directly impact the course of letter processing.

The finding that letter names are involved in the mediation of decontextualized letters' identicalness when their processing requires going beyond a perceptual level does not necessarily mean that readers do not have available other types of letter representations that, if necessary, may take on such a mediating function. In particular, individuals who have lost the ability to access letters' names due to neurological injuries (see, e.g., Mycroft et al., 2002) are likely to compensate for such a deficiency via enhanced reliance on nonnominal letter representations. Interestingly, the markedly reduced syllabic-length effects found with increases in letter-processing speed suggest that - as a result of enhanced automation- regular readers, too, may shift from a predominantly nominal letter-processing strategy to increased reliance on alternative nonnominal letter representations. However, given that, such a decrease notwithstanding, bias from syllabic length on processing time remained remarkably robust, the conclusion that-at least for regularly developing Hebrew readers-the retrieval of nominal representations continues to be an automatic response to the encounter of decontextualized letters as processing proceeds beyond a perceptual level seems warranted.

Note, finally, that the present study was conducted with Hebrew readers/writers who, as novice readers, had been taught to refer to letters by their names rather than by their sounds. This may reasonably explain why in the present study, letter names were found to carry a mediating function in the processing of isolated letters. Whether learning letters initially in conjunction with their sounds rather than with their names (see, e.g., Levin et al., 2005) would have led to an essential change in the strategy used for processing isolated letters cannot be determined by our findings and should be further clarified in future research. Until then, some caution should be applied in generalizing the conclusions from the present study.

Author note This manuscript was prepared with the support of the Science of Learning Center on Visual Language and Visual Learning (VL2), National Science Foundation Grant SBE-0541953. The analyzed data were collected within an international reading project executed in collaboration with Tevhide Kargin (Ankara University), Christian Rathmann (Hamburg University), and Peter Hauser (Rochester Institute of Technology). 
Appendix A

Table 3 Letter stimulus pairs used in the P-and C-conditions

\begin{tabular}{|c|c|c|c|c|c|c|c|c|}
\hline & \multicolumn{4}{|c|}{ P-Condition } & \multicolumn{4}{|c|}{ C-Condition } \\
\hline & \multicolumn{2}{|c|}{ YES } & \multicolumn{2}{|c|}{ NO } & \multicolumn{2}{|c|}{ YES } & \multicolumn{2}{|c|}{ NO } \\
\hline \multirow{10}{*}{ 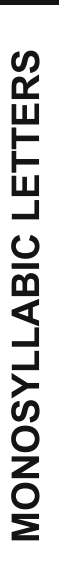 } & ב & 2 & 9 & 0 & 2 & ค & 9 & 0 \\
\hline & ש & ש & $n$ & 2 & ש & $e$ & $n$ & D \\
\hline & 0 & v & ט & ש & v & 0 & 0 & $e$ \\
\hline & 9 & פ & ב & $n$ & 9 & 0 & ב & $N$ \\
\hline & $n$ & $n$ & פ & ש & $n$ & $N$ & 9 & $e$ \\
\hline & ค & ค & 0 & 0 & D & 2 & 0 & 9 \\
\hline & $e$ & $e$ & $N$ & م & $e$ & ש & ค & $n$ \\
\hline & 0 & 0 & 6 & $e$ & 0 & v & $e$ & 0 \\
\hline & 0 & 0 & ค & $N$ & 0 & 9 & $N$ & 2 \\
\hline & $N$ & $N$ & 0 & $e$ & $N$ & $n$ & 0 & ש \\
\hline \multirow{10}{*}{ 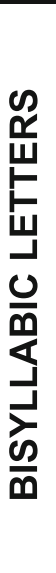 } & $\lambda$ & $\lambda$ & $\mathbf{Y}$ & $\lambda$ & $\lambda$ & $d$ & $\mathbf{Y}$ & $d$ \\
\hline & $\mathbf{y}$ & $\mathbf{Y}$ & T & א & $\mathbf{y}$ & 3 & $T$ & $k$ \\
\hline & 4 & 4 & 4 & $\lambda$ & 4 & $f$ & 4 & $d$ \\
\hline & $\boldsymbol{x}$ & $\boldsymbol{K}$ & $\boldsymbol{x}$ & $\mathbf{T}$ & א & $k$ & $\boldsymbol{x}$ & 3 \\
\hline & $\mathbf{T}$ & $\boldsymbol{T}$ & 4 & $\mathbf{y}$ & $\mathbf{T}$ & 3 & 4 & 3 \\
\hline & $d$ & $d$ & 3 & $c$ & $d$ & $\lambda$ & 3 & $\lambda$ \\
\hline & 3 & 3 & 3 & $k$ & 3 & $\mathbf{y}$ & ? & א \\
\hline & $f$ & $f$ & $f$ & $d$ & $f$ & ל & $d$ & ל \\
\hline & $k$ & $k$ & $k$ & ? & $k$ & א & $k$ & T \\
\hline & 3 & 3 & $f$ & 3 & 3 & $\boldsymbol{T}$ & 3 & 4 \\
\hline
\end{tabular}

Pairs designed to elicit a "no" or a "yes" response are shown for each condition.

Table 4 The letters of the Hebrew alphabet

\begin{tabular}{|c|c|c|c|c|c|c|c|c|c|c|c|c|c|c|c|c|c|c|c|c|c|c|}
\hline Print & ת & ש & 7 & ק & $\mathbf{Y}$ & 9 & $y$ & 0 & נ & $n$ & 4 & כ & I & 0 & $\mathbf{n}$ & $T$ & I & ה & $\mathbf{T}$ & $\lambda$ & 2 & $\boldsymbol{K}$ \\
\hline Cursive & $n$ & $e$ & ? & $p$ & 3 & 0 & $\gamma$ & 0 & $J$ & $N$ & $f$ & 3 & 1 & 6 & $n$ & 3 & 1 & ת & 3 & $d$ & D & $k$ \\
\hline
\end{tabular}




\section{Appendix B}

Table 5 Percentile occurrence of monosyllabic letter stimuli within 8,841 words

\begin{tabular}{|c|c|c|c|c|c|c|}
\hline \multirow{2}{*}{$\begin{array}{l} \\
\text { Syllabic } \\
\text { Length }\end{array}$} & Text Type & \multicolumn{3}{|c|}{ School Textbooks } & \multirow{2}{*}{\begin{tabular}{|c|}
$\begin{array}{c}\text { Internet } \\
\text { Articles }\end{array}$ \\
For adults
\end{tabular}} & \multirow{2}{*}{$\begin{array}{l}\text { All Texts } \\
\text { Overall }\end{array}$} \\
\hline & Letters & $\begin{array}{c}\text { Grade } \\
3-4 \\
\end{array}$ & $\begin{array}{c}\text { Grade } \\
6-7 \\
\end{array}$ & $\begin{array}{c}\text { Grade } \\
9-10 \\
\end{array}$ & & \\
\hline \multirow{6}{*}{ 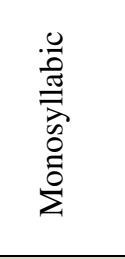 } & ב (Bet) & 4.7 & 4.8 & 4.3 & 5.1 & 4.7 \\
\hline & v (Tet) & 1.1 & 1.1 & 1.0 & 1.3 & 1.1 \\
\hline & מ (Mem) (M) & 5.3 & 6.0 & 5.3 & 4.3 & 5.2 \\
\hline & (Pei) & 1.2 & 1.5 & 1.8 & 1.8 & 1.6 \\
\hline & $w$ (Shin) $\quad$ (Sh) & 4.6 & 4.7 & 3.7 & 4.3 & 4.3 \\
\hline & All letters & 3.4 & 3.6 & 3.2 & 3.3 & 3.4 \\
\hline \multirow{6}{*}{ 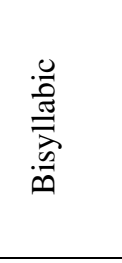 } & $\kappa($ Aleph) (A) & 4.1 & 4.7 & 3.9 & 5.0 & 4.4 \\
\hline & $\lambda$ (Gimel) $\quad(\mathrm{G})$ & 1.0 & 1.6 & 1.4 & 1.4 & 1.3 \\
\hline & $T$ (Dalet) $\quad(\mathrm{D})$ & 1.6 & 2.5 & 2.2 & 2.4 & 2.1 \\
\hline & $\zeta$ (Lamed) (L) & 6.8 & 6.7 & 6.5 & 6.3 & 6.6 \\
\hline & צ (Tzadik) (TS) & 1.1 & 1.2 & 1.2 & 1.3 & 1.2 \\
\hline & All letters & 2.9 & 3.3 & 3.3 & 3.3 & 3.4 \\
\hline
\end{tabular}

\section{References}

Arguin, M., \& Bub, D. N. (1995). Visual word activation in pure alexia. Brain and Language, 49, 77-103.

Blake, R. R., Fox, R., \& Lappin, J. S. (1970). An invariance in the reaction time classification of same and different letter pairs. Journal of Experimental Psychology, 85, 133-137. doi:10.1037/ h0029520

Boles, D. B., \& Eveland, D. C. (1983). Visual and phonetic codes and the process of generation in letter matching. Journal of Experimental Psychology: Human Perception and Performance, 9, 657-674.

Carrasco, M., Kinchla, R. A., \& Figueroa, J. G. (1988). Visual lettermatching and the time course of visual and acoustic codes. Acta Psychologica, 69, 1-17.

Coltheart, M. (1999). Modularity and cognition. Trends in Cognitive Sciences, 3, 115-120.

Coslett, H. B., \& Saffran, E. M. (1992). Optic aphasia and the right hemisphere: A replication and extension. Brain and Language, 43, 148-161.

Dainoff, M. J. (1970). Time course of visual and auditory encoding. Journal of Experimental Psychology, 86, 214-224.

Dainoff, M., \& Haber, R. N. (1970). Effect of acoustic confusability on levels of information processing. Canadian Journal of Psychology, 24, 98-108. doi:10.1037/h0082847
Ellefson, M. R., Treiman, R., \& Kessler, B. (2009). Learning to label letters by sounds or names: A comparison of England and the United States. Journal of Experimental Child Psychology, 102, 323-341. doi:10.1016/j.jecp.2008.05.008

Finkbeiner, M., \& Coltheart, M. (2009). Letter recognition: From perception to representation. Cognitive Neuropsychology, 26, 1-6. doi:10.1080/02643290902905294

Forster, K. I., \& Forster, J. C. (2003). DMDX: A Windows display program with millisecond accuracy. Behavior Research Methods, Instruments, \& Computers, 35, 116-124. doi:10.3758/ BF03195503

Grainger, J., \& Jacobs, A. M. (1996). Orthographic processing in visual word recognition: A multiple read-out model. Psychological Review, 103, 518-565. doi:10.1037/0033-295X.103.3.518

Jackson, N., \& Coltheart, M. (2001). Routes to reading success and failure. Hove, U.K.: Psychology Press.

James, T. W., \& Cree, G. S. (2010). Perceptual and conceptual interactions in object recognition and expertise. In I. Gauthier, M. J. Tarr, \& D. Bub (Eds.), Perceptual expertise: Bridging brain and behavior (pp. 333-352). New York, NY: Oxford University Press.

James, K. H., Wong, A. C.-N., \& Jobard, G. (2010). The case for letter expertise. In I. Gauthier, M. J. Tarr, \& D. Bub (Eds.), Perceptual expertise: Bridging brain and behavior (pp. 305-332). New York, NY: Oxford University Press. 
Kinoshita, S., \& Kaplan, L. (2008). Priming of abstract letter identities in the letter match task. Quarterly Journal of Experimental Psychology, 61, 1873-1885. doi:10.1080/17470210701781114

Levin, I. (2007). The role of Hebrew letter names in early literacy: The case of multiphonemic acrophonic names. Journal of Experimental Child Psychology, 98, 193-216.

Levin, I., \& Aram, D. (2004). Children's names contribute to early literacy: A linguistic and a social perspective (pp. 219-241). In D. Ravid \& H. Bat-Zeev Shyldkrot (Eds.), Perspectives on language and language development. Dordrecht, The Netherlands: Kluwer.

Levin, I., Shatil-Carmon, S., \& Asif-Rave, O. (2005). Learning of letter names and sounds and contribution to word reading. Journal of Experimental Child Psychology, 93, 139-165.

Miller, P. (2005). Reading Experience and changes in the processing of letters, written words, and pseudo-homophones: Comparing fifthgrade students and university students. Journal of Genetic Psychology, 164, 407-434.

Miozzo, M., \& Caramazza, A. (1998). The varieties of pure alexia: The case of failure to access graphemic representations. Cognitive Neuropsychology, 15, 203-30.

Mycroft, R., Hanley, J. R., \& Kay, J. (2002). Preserved access to abstract letter identities despite abolished letter naming in a case of pure alexia. Journal of Neurolinguisitcs, 15, 99-108.

Posner, M. I., \& Keele, S. W. (1967). Decay of visual information from a single letter. Science, 158, 137-139. doi:10.1126/science.158.3797.137

Posner, M. I., \& Mitchell, R. F. (1967). Chronometric analysis of classification. Psychological Review, 74, 392-409. doi:10.1037/ h0024913
Proctor, R. W. (1981). A unified theory for matching-task phenomena. Psychological Review, 88, 291-326. doi:10.1037/0033295X.88.4.291

Raynard, D., \& Besner, D. (1987). Basic processes in reading: On the development of cross-case letter matching without reference to phonology. Bulletin of the Psychonomic Society, 25, 361-368.

Reitsma, P. (1978). Changes in letter processing in beginning readers. Journal of Experimental Child Psychology, 25, 315-325.

Rittle-Johnson, R. B., \& Siegler, R. S. (1999). Learning to spell: Variability, choice, and change in strategy use. Child Development, 70, 332-348.

Saffran, E. M., \& Coslett, H. B. (1998). Implicit vs. letter-by-letter reading in pure alexia: A tale of two systems. Cognitive Neuropsychology, 15, 141-165. doi:10.1080/026432998381249

Soler, M. J., \& Salvador, A. (1988). Same-different discrepancy in exhaustive matching tasks. Bulletin of the Psychonomic Society, 26, 209-211.

Thorson, G., Hochhaus, L., \& Stanners, R. F. (1976). Temporal changes in visual and acoustic codes in a letter-matching task. Perception \& Psychophysics, 19, 346-348.

Treiman, R., Levin, I., \& Kessler, B. (2006). Learning of letter names follows similar principles across languages: Evidence from Hebrew. Journal of Experimental Child Psychology, 27, 211-227.

Treiman, R., \& Rodriguez, K. (1999). Young children use letter names in learning to read words. Psychological Science, 10, 334-338.

Treiman, R., Sotak, L., \& Bowman, M. (2001). The role of letter names and letter sounds in connecting print and speech. Memory \& Cognition, 29, 860-873. 